\title{
Jamming Resistance of the Inbound Channel of an Identification System with Broadband Signals and Error Control Codes in the Conditions of Pulse Noise and Intra-System Jamming
}

Sergey B. Zhironkin, Alexander A. Bliznyuk and Alexander A. Kuchin* Military Academy of Aero-Space Defense named after the Marshal of Soviet Union G.K. Zhukov 50 Zhigareva Str., Tver, 170100, Russia

Received 25.02.2019, received in revised form 01.04.2019, accepted 23.04.2019

Results of an assessment of a noise stability of the request channel of a radar-tracking identification with broadband signals in the conditions of pulse noise and intersystem hindrances are given. Optimum alarm and code designs of orthogonal broadband signals decide on Read-Solomon's correcting codes for various characteristics of influencing hindrances.

Keywords: jamming resistance, the worst jamming, broadband signals, signal-code construction.

Citation: Zhironkin S.B., Bliznyuk A.A., Kuchin A.A. Jamming resistance of the inbound channel of an identification system with broadband signals and error control codes in the conditions of pulse noise and intra-system jamming, J. Sib. Fed. Univ. Eng. technol., 2019, 12(6), 673-682. DOI: 10.17516/1999-494X-0166.

\section{Помехоустойчивость запросного канала опознавания}

\section{с широкополосными сигналами и корректирующими кодами \\ в условиях импульсных шумовых и внутрисистемных помех}

\author{
С.Б. Жиронкин, А.А. Близнюк, А.А. Кучин \\ Военная академия воздушно-космической оборонь \\ им. Маршала Советского Союза Г.К. Жукова \\ Россия, 170100, Тверь, ул. Жигарева, 50
}

Приводятся результаты оченки помехоустойчивости запросного канала радиолокационного опознавания с широкополосными сигналами в условиях импульсных шумовых и внутрисистемных

(C) Siberian Federal University. All rights reserved

This work is licensed under a Creative Commons Attribution-NonCommercial 4.0 International License (CC BY-NC 4.0).

* Corresponding author E-mail address: kuchin.a.a@gmail.com, iadrin@mail.ru 
помех. Определяются оптимальные сигнально-кодовые конструкции ортогональных широкополосных сигналов с корректирующими кодами Рида-Соломона для различньх характеристик воздействующих помех.

Ключевые слова: помехоустойчивость, наихудшая помеха, иирокополоснье сигналь, сигнальнокодовая конструкция.

\section{Введение}

Применение широкополосных сигналов в средствах системы радиолокационного опознавания способно улучшить их информационные характеристики в наиболее сложных условиях радиоэлектронного конфликта: воздействие помех, разведка и имитация сигналов и пр. [1, 2]. Современные методы радиоэлектронного противодействия позволяют создавать помехи, оптимизированные к характеристикам подавляемых средств, например импульсные шумовые помехи, сигналоподобные помехи и пр. [3-5], что следует учитывать при обосновании параметров широкополосных сигналов опознавания. Кроме того, при работе средств опознавания в общих зонах создаются большие потоки внутрисистемных помех (ВСП), которые необходимо рассматривать в комплексе с преднамеренными и с учетом специфики каналов опознавания.

Цель работы - оценка помехоустойчивости запросного канала системы радиолокационного опознавания с широкополосными ортогональными сигналами (ШПС) в условиях воздействия импульсных шумовых и внутрисистемных помех и оптимальных для этих условий параметров корректирующих кодов Рида-Соломона (РС).

\section{Постановка задачи}

Для достижения поставленной цели введем следующие ограничения и допущения.

1. Рассматриваются запросные сигналы опознавания, информационная часть которых содержит $I$ недвоичных импульсов для передачи $m$ бит информации с учетом требований к защите ответчиков от имитации сигналов методом «наугад», предъявляемых к средствам современной системы [1].

2. Сигнально-кодовые конструкции запросного сигнала формируются на основе комбинаций ансамбля из $M<2^{m}$ ортогональных сигналов с кодами Рида-Соломона, корректирующими ошибки.

3. Внутрисистемные помехи создаются большим количеством $\left(N_{s}>1\right)$ взаимодействующих в общей зоне запросчиков с одинаковыми характеристики. Несинхронный поток широкополосных сигналов-помех является простейшим.

4. Показателем помехоустойчивости запросного канала служит вероятность $P$ правильного приема информационной части запросного сигнала, состоящей из $m$ бит:

$$
P=\left(1-P_{e}\right)^{I},
$$

где $P_{e}-$ вероятность ошибки при различении $M$ детерминированных ортогональных сигналов; $I$ - число недвоичных импульсов в запросном сигнале, необходимых для передачи $m$ бит, при заданном отношении сигнал/помеха.

Для сравнительной оценки последовательно рассмотрим вклад различных составляющих воздействующих на запросный канал (на вход ответчика) помех в ошибки опознавания.

$$
-674-
$$




\section{Основная часть}

Вероятность ошибки при различении $M$ детерминированных ортогональных сигналов при действии белого гауссова шума (БГШ) с односторонней спектральной плотностью мощности $N_{j}$ определяется выражением [6]

$$
P_{e}=1-\frac{1}{\sqrt{2 \pi}} \int_{-\infty}^{\infty} \exp \left[-\frac{1}{2}\left(x-\sqrt{\frac{2 m E_{b}}{I N_{j}}}\right)^{2}\right] \Phi^{M-1}(x) d x,
$$

где $E_{b}$ - энергия сигнала, приходящаяся на один бит передаваемой информации, $\Phi(x)=\frac{1}{\sqrt{2 \pi}} \int_{-\infty}^{x} \exp \left(-\frac{t^{2}}{2}\right) d t-$ интеграл вероятности.

Для каналов обмена данными с ШПС с внутриимпульсной манипуляцией особую опасность, как и в [3], представляет прерывистая (импульсная) шумовая помеха с постоянным значением средней по времени спектральной плотности мощности, являющая собой случайный поток импульсов БГШ $[3,5]$. Такую помеху с оптимальным для подавления значением средней скважности называют наихудшей $[7,8]$. При излучении импульсной шумовой помехи ее односторонняя спектральная плотность мощности равна $N_{j} / \rho$, где $N_{j}$ - средняя по времени спектральная плотность мощности, $\rho$ - величина, обратная средней скважности помехи.

С учетом введенной характеристики помехи вероятность ошибки при ее действии может быть записана в виде

$$
P_{e}(\rho)=\rho\left[1-\frac{1}{\sqrt{2 \pi}} \int_{-\infty}^{\infty} \exp \left[-\frac{1}{2}\left(x-\sqrt{\frac{2 m E_{b} \rho}{I N_{j}}}\right)^{2}\right] \Phi^{M-1}(x) d x\right] .
$$

Помеха с параметром $\rho=\rho^{*}$, максимизирующим вероятность ошибки, наихудшая. Значение $\rho^{*}$ и соответствующее ему значение максимальной вероятности ошибки $P_{e \_ \text {max }}$ при действии наихудших импульсных шумовых помех определяются численно и могут быть рассчитаны с использованием оценочных коэффициентов $C_{M}$ и $D_{M}[9]$ :

$$
\begin{aligned}
& \rho^{*}=\left\{\begin{array}{l}
1, E / N_{j}<C_{M}, \\
\frac{C_{M}}{E / N_{j}}, E / N_{j} \geq C_{M},
\end{array}\right. \\
& P_{e_{-} \max }=\left\{\begin{array}{l}
P_{e}(1), E / N_{j}<C_{M}, \\
\frac{D_{M}}{E / N_{j}}, E / N_{j} \geq C_{M},
\end{array}\right.
\end{aligned}
$$

где $E=\frac{m E_{b}}{I}-$ энергия импульса.

В таблице в качестве примера для $M=2-1024$ приведены значения таких коэффициентов $[7,10]$.

Результаты расчетов помехоустойчивости запросного канала широкополосной системы опознавания в условиях наихудшей шумовой помехи при $m=44$ бит, $M=256$ представлены на 
Таблица. Значение коэффициентов $\mathrm{C}_{\mathrm{M}}$, $\mathrm{D}_{\mathrm{M}}$ для различных $\mathrm{M}$

Table. The value of the coefficients $C_{M}, D_{M}$ for different $M$

\begin{tabular}{|c|c|c|}
\hline$M$ & $C_{M}$ & $D_{M}$ \\
\hline 2 & 1,4176 & 0,1657 \\
\hline 4 & 1,7010 & 0,3589 \\
\hline 8 & 2,0116 & 0,5780 \\
\hline 16 & 2,3451 & 0,8212 \\
\hline 32 & 2,6987 & 1,0861 \\
\hline 64 & 3,0695 & 1,3704 \\
\hline 128 & 3,3396 & 1,6698 \\
\hline 256 & 3,9746 & 1,9873 \\
\hline 512 & 4,3282 & 2,3187 \\
\hline 1024 & 4,6150 & 2,6615 \\
\hline
\end{tabular}

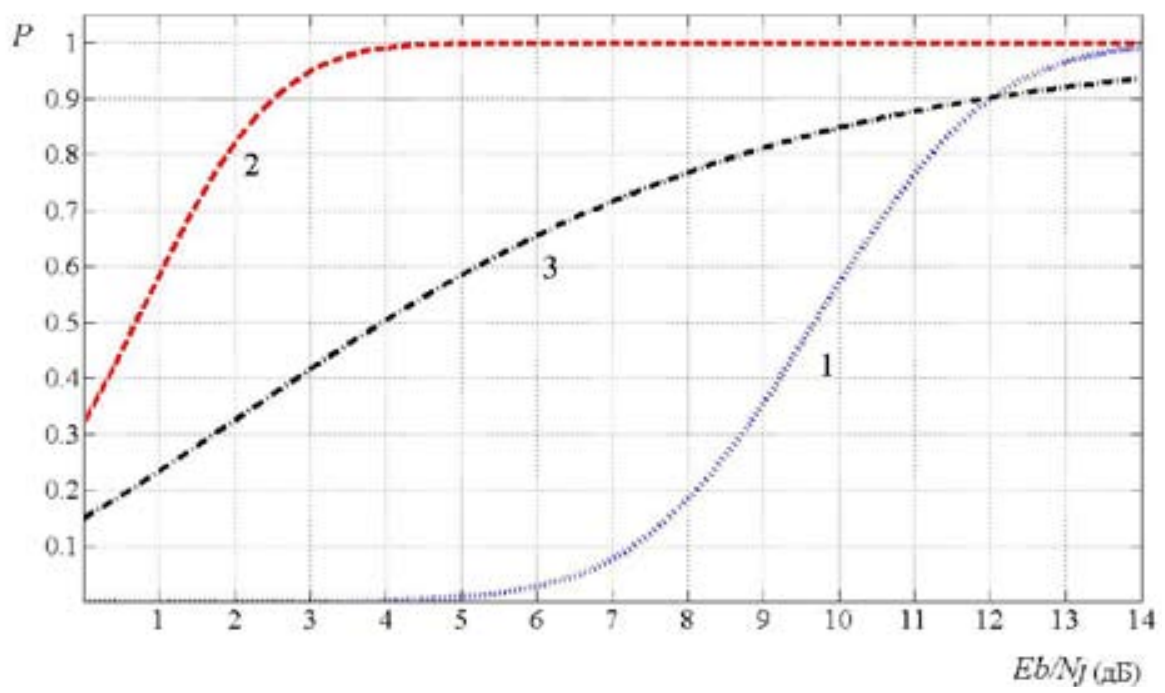

Рис. 1. Помехоустойчивость запросного канала широкополосной системы опознавания: 1 - двоичный сигнал с амплитудной манипуляцией; 2 - недвоичный ШПС для $M=256$ при действии непрерывной помехи; 3 - недвоичный ШПС для $M=256$ при действии наихудшей импульсной помехи

Fig. 1. Interference immunity of the inquiring channel of the broadband identification system: 1 - a binary signal with amplitude shift keying; 2 - non-binary broadband signal for $M=256$ under the action of continuous interference; 3 - non-binary broadband signal for $M=256$ under the action of the worst impulse noise

рис. 1 кривой 3. Для сравнения также изображены кривые: 1 - двоичный сигнал с амплитудной манипуляцией (ИВК); 2 - недвоичный ШПС для $M=256$ при действии непрерывной помехи.

При расчете для двоичного ИВК современной системы опознавания [1] использовали соотношение (1), где вместо $I$ подставлялось $m=44$ и вероятность ошибочного приема [6]

$$
P_{e}=1-\Phi\left(\sqrt{\frac{E_{b}}{2 N_{0}}}\right) .
$$


Для недвоичного ШПС при действии непрерывной шумовой помехи использовали (1) и (2) при $\rho=1$.

Рисунок 1 демонстрирует, что на уровне $P=0,9$ применение недвоичных ортогональных ШПС обеспечивает энергетический выигрыш в помехоустойчивости по сравнению с ИВК около 10 дБ при действии непрерывной помехи. Однако при действии наихудшей импульсной шумовой помехи этот выигрыш сводится к нулю.

Для сохранения высокой помехоустойчивости каналов с ШПС при действии наихудших импульсных шумовых помех и большом количестве взаимодействующих средств опознавания применяют избыточное помехоустойчивое кодирование [9], например блоковые коды с максимальным расстоянием. К ним относятся избыточные коды Рида-Соломона [1]. Основными параметрами блоковых $(n, k, t)$-кодов являются: $n$ - общее число символов (недвоичных импульсов) в блоке кода, $k$ - число информационных символов в блоке кода $\left(k \cdot \log _{2} M \geq m\right), t$ - число исправляемых ошибок в блоке кода. Для кодов РС параметры $n, k, t$ связаны соотношением $n=k+2 t$, где $n=M-1$, а для укороченных кодов можно принимать любое меньшее значение.

Для случая совместного применения недвоичных ортогональных сигналов и избыточных кодов РС использовалось соотношение (2), в которое вместо $I$ подставляли $n$, и

$$
P=\sum_{i=0}^{t} C_{n}^{i} P_{e}^{i}\left(1-P_{e}\right)^{n-i},
$$

где $C_{n}^{i}$ - число сочетаний из $n$ элементов по $i$.

Результаты расчетов иллюстрирует рис. 2, который наглядно демонстрирует, что энергетический проигрыш при действии наихудшей импульсной шумовой помехи может быть минимизирован выбором СКК.

Нахождение СКК, обеспечивающей наиболее высокую помехоустойчивость, иллюстрируется графиками на рис. 3, 4. Критерием выбора наиболее энергетически эффективной СКК является минимально требуемое $\left(E_{b} / N_{j}\right)_{\text {треб }}^{(\min )}$ отношение энергии принимаемого приемником средства опознавания сигнала (в расчете на один бит передаваемой информации) к спектральной плотности мощности помехи для достижения заданной вероятности правильной доставки информационной части запросного сигнала $P=0,9$.

Полученные результаты позволяют определять СКК для запросного канала системы радиолокационного опознавания с ШПС, обеспечивающие наибольшую помехоустойчивость относительно наихудших импульсных шумовых помех.

Реально прием сигналов опознавания будет осуществляться на фоне комплекса помех, включающего преднамеренные и внутрисистемные помехи, а также собственный шум приемника. При принятых условиях и ограничениях в качестве модели взаимной (внутрисистемной) помехи в системе опознавания с ШПС может рассматриваться непрерывный нормальный шум (БГШ) со спектральной плотностью мощности $N_{0}$ [11], включающий и собственный шум приемника ответчика. В этом случае воздействующая помеха сводится к сумме непрерывной и импульсной шумовых помех и на входе приемника наблюдается отношение сигнал/суммарная помеха.

$$
-677-
$$




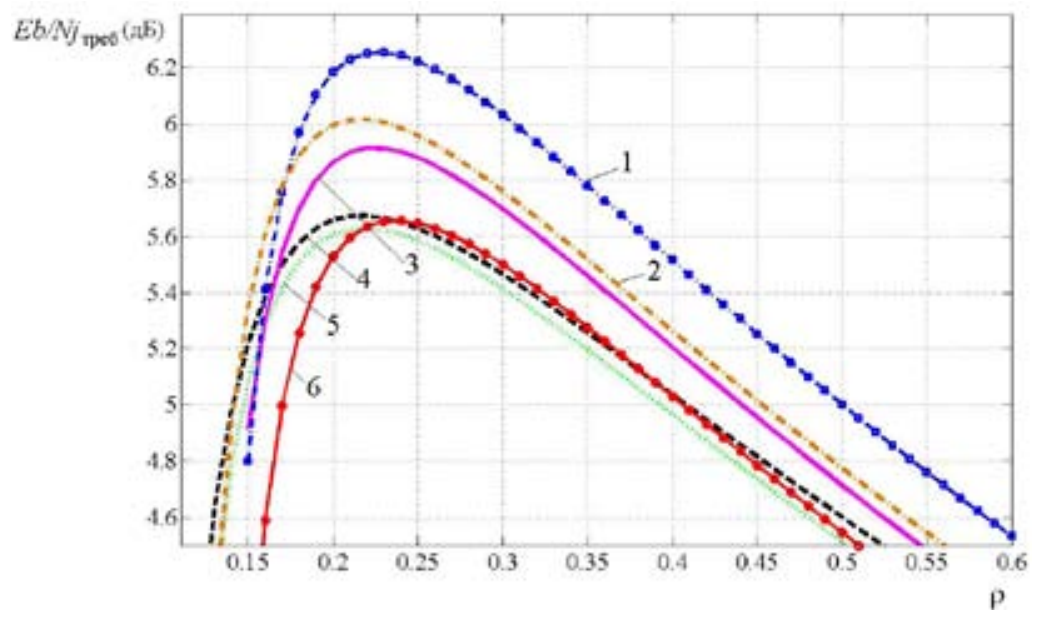

Рис. 2. Зависимость отношения сигнал/помеха, требуемого для обеспечения вероятности правильного приема информационной части запросного сигнала $P=0,9$, от $\rho$ для $M=16-1024: 1$ - 1024-ный ортогональный ШПС с РС-кодом (9,5,2); 2 - 128-ный ортогональный ШПС с РС-кодом (11,7,2); 3 - 256-ный ортогональный ШПС с РС-кодом $(10,6,2) ; 4$ - 32-ный ортогональный ШПС с РС-кодом $(13,9,2) ; 5$ - 16-ный ортогональный ШПС с РС-кодом $(15,11,2) ; 6$ - 512-ный ортогональный сигнал с РС-кодом $(9,5,2)$

Fig. 2. Dependence of the signal / interference ratio required to ensure the probability of correct reception of the information part of the request signal $\mathrm{P}=0.9$, from $\mathrm{r}$ for $M=16-1024$ : 1 - 1024-ary orthogonal broadband signal with a RS code (9.5.2); 2 - 128-ary orthogonal broadband signal with a RS code (11,7,2); 3 - 256-ary orthogonal broadband signal with a RS code $(10,6,2)$; 4 - 32-ay orthogonal broadband signal with a RS code $(13,9,2) ; 5$ - 16 day orthogonal wideband signal with a RS code $(15,11,2) ; 6$ - 512-ary orthogonal broadband signal with a RS code $(9,5,2)$

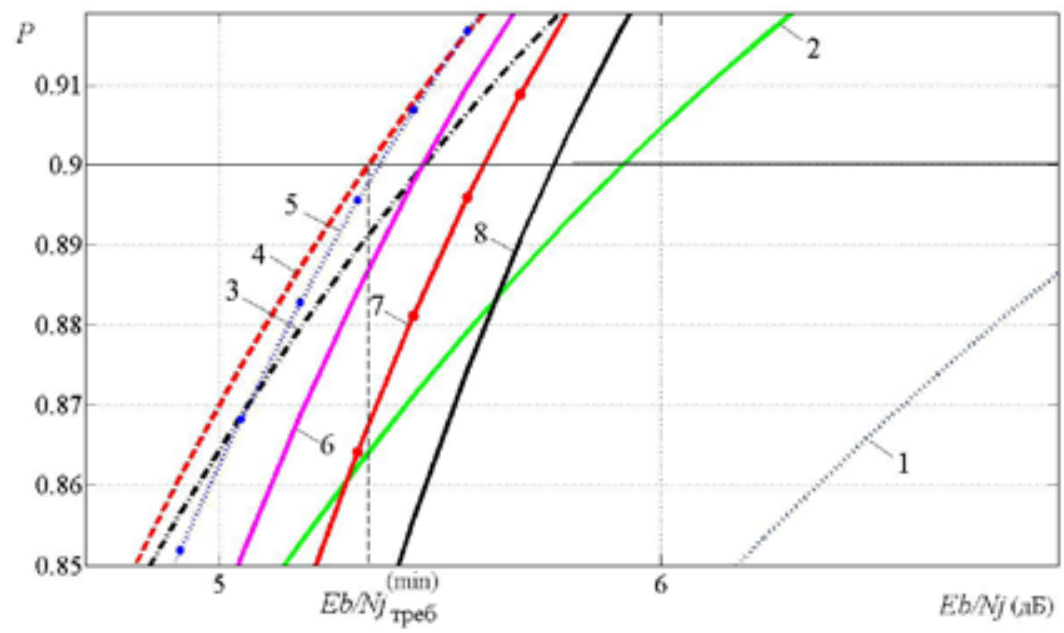

Рис. 3. Вероятность правильного приема информационной части запросного сигнала для недвоичного ортогонального ШПС с различными кодами Рида-Соломона при действии наихудшей импульсной помехи: 1 - РС-код (8,6,1); 2 - РС-код (10,6,2); 3 - РС-код (12,6,3); 4 - РС-код $(14,6,4) ; 5$ - РС-код $(16,6,5)$; 6 - РС-код $(18,6,6) ; 7$ - РС-код $(20,6,7) ;$ РС-код $(22,6,8)$

Fig. 3. The probability of correct reception of the information part of the request signal for a non-binary orthogonal broadband signal with different Reed-Solomon codes under the effect of the worst impulse noise: 1 - RS code $(8,6,1) ; 2$ - RS code $(10,6,2) ; 3$ - RS code $(12,6,3) ; 4$ - RS code $(14,6,4) ; 5$ - RS code $(16.6 .5) ; 6$ - RS code $(18,6,6)$; 7 - RS code $(20,6,7)$; RS code $(22,6,8)$ 


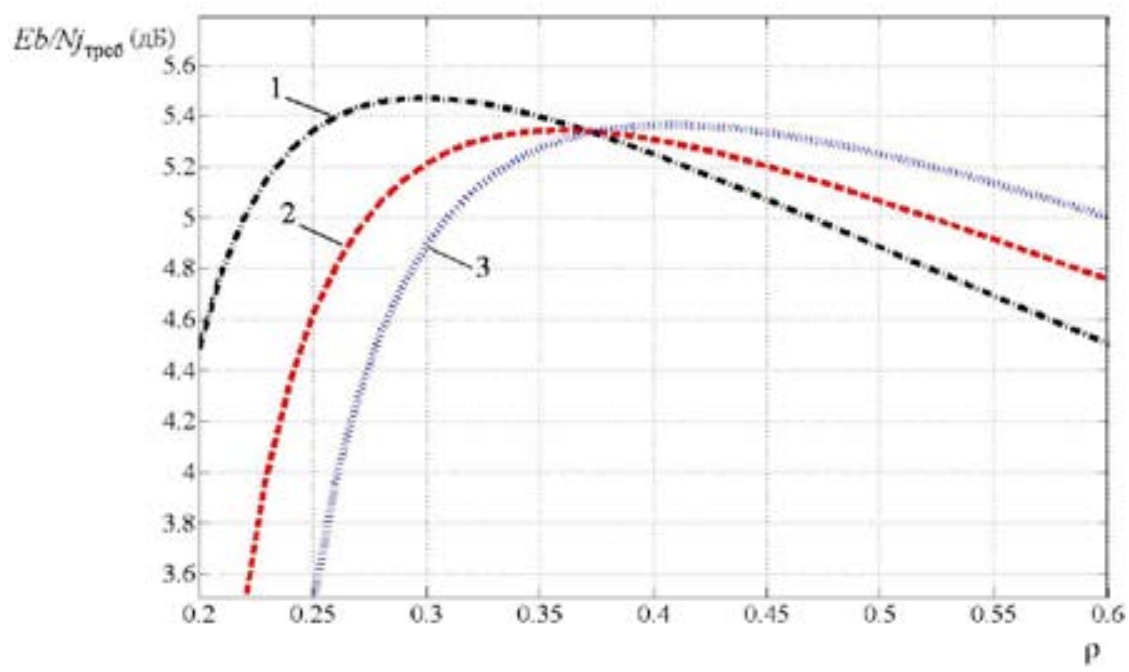

Рис. 4. Требуемое отношение сигнал/помеха для достижения заданной вероятности правильного приема информационной части запросного сигнала $P=0,9$ для $M=256: 1-256$-ный ортогональный ШПС с РСкодом (12,6,3); 2 - 256-ный ортогональный ШПС с РС-кодом (14,6,4); 3 - 256-ный ортогональный ШПС с РС-кодом $(16,6,5)$

Fig. 4. The required signal-to-noise ratio to achieve a given probability of correct reception of the information part of the request signal is $\mathrm{P}=0.9$ for $\mathrm{M}=256$ : 1 - 256-ary orthogonal broadband signal with a $\mathrm{RS}$ code (12.6.3); 2 - 256-ary orthogonal broadband signal with a RS code $(14,6,4) ; 3$ - 256-ary orthogonal broadband signal with a RS code $(16.6,5)$

$$
\frac{E_{b}}{N_{j 0}}=\frac{E_{b}}{N_{j}+N_{0}}=\frac{1}{\left[\left(\frac{E_{b}}{N_{j}}\right)^{-1}+\left(\frac{E_{b}}{N_{0}}\right)^{-1}\right]} .
$$

Спектральная плотность шумовой помехи эквивалентна внутрисистемной помехе, может быть определена по формуле

$$
N_{0}=n_{3} \cdot \frac{P_{s}}{F},
$$

где $n_{3}$ - среднее число одновременно работающих с ответчиком запросчиков; $P_{s}-$ мощность сигналов ВСП от каждого из запросчиков на входе приемника; $F$ - ширина спектра ШПС.

Вероятность ошибочного приема недвоичного импульса для рассматриваемого случая определяется соотношением

$$
\begin{aligned}
& P_{e}(\rho)=\rho\left[1-\frac{1}{\sqrt{2 \pi}} \int_{-\infty}^{\infty} \exp \left[-\frac{1}{2}\left(x-\sqrt{\frac{2 m E_{b}}{n\left(\left(N_{j} / \rho\right)+N_{0}\right)}}\right)^{2}\right] \Phi^{M-1}(x) d x\right]+ \\
& +(1-\rho)\left[1-\frac{1}{\sqrt{2 \pi}} \int_{-\infty}^{\infty} \exp \left[-\frac{1}{2}\left(x-\sqrt{\frac{2 m E_{b}}{n N_{0}}}\right)^{2}\right] \Phi^{M-1}(x) d x\right] .
\end{aligned}
$$

Требуемые значения сигнал/помеха представлены на рис. 5.

Уменьшение значений отношения сигнал/непрерывная шумовая помеха $E_{b} / N_{0}$ (рис. 5), т.е. увеличение мощности непрерывной помехи, приводит к изменению значения $\rho^{*}$ наихудшей 


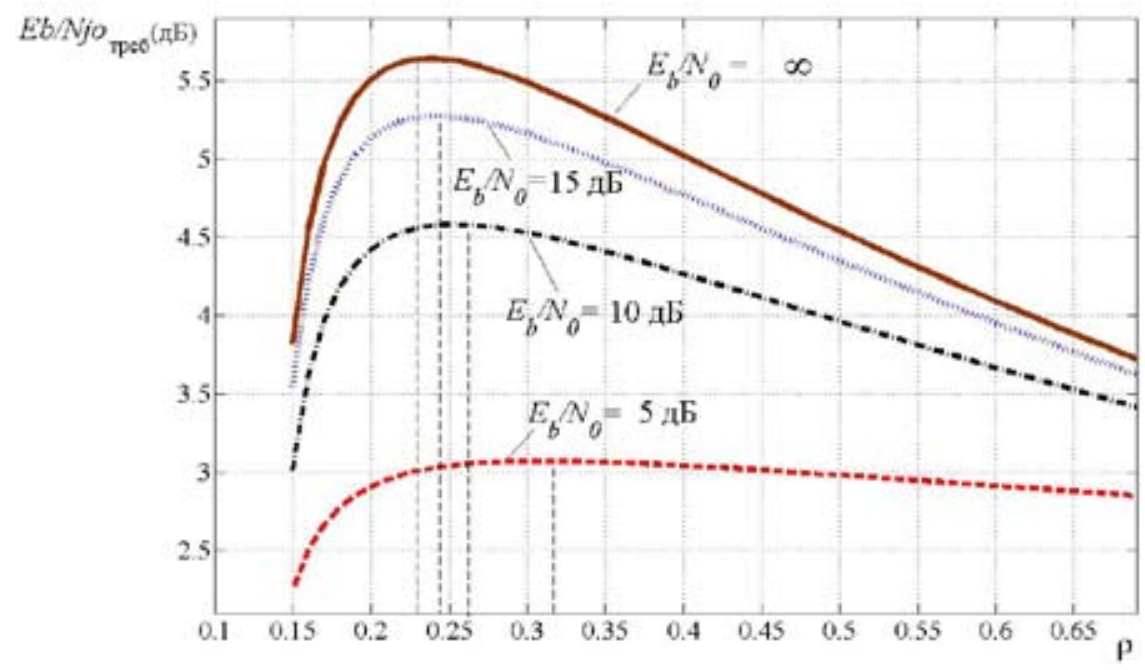

Рис. 5. Требуемое отношение сигнал/помеха для достижения заданной вероятности правильного приема информационной части запросного сигнала $P=0,9$ при различных отношениях $E_{b} / N_{0}$ для СКК $M=512 \mathrm{c}$ РС-кодом $(9,5,2)$ при действии на канал опознавания комплекса помех

Fig. 5. Required signal-to-noise ratio to achieve a given probability of correct reception of the information part of the request signal $P=0.9$ for various $E_{b} / N_{0}$ ratios for SCD $M=512$ with a RS code (9.5.2), when acting on the identification channel interference complex

шумовой помехи и, соответственно, к уменьшению требуемого отношения сигнал/суммарная помеха $E_{b} / N_{j 0}$.

Оценка состава и параметров действующих помех позволяет оптимизировать прием сигналов к складывающимся условиям и обосновывать необходимые для этого отношения сигнал/ помеха.

Различный состав и характеристики воздействующих помех приводят к изменению выбора СКК. Так, например, для условий воздействия только импульсной шумовой помехи наилучшей является СКК $M=32$ с РС-кодом $(21,9,6)$. При одновременном действии с ней непрерывной шумовой помехи (ВСП), при $E_{b} / N_{0}=5$ дБ, наилучшей становится СКК $M=32$ с РС-кодом $(15,9,3)$, а СКК $M=512$ с РС-кодом $(9,5,2)$ по помехоустойчивости непосредственно приближается к ней.

\section{Заключение}

Таким образом, полученные результаты оценки помехоустойчивости запросного канала опознавания с $M$-ными ортогональными широкополосными сигналами и кодами РидаСоломона, корректирующими ошибки, показали их оптимальность в условиях воздействия импульсных шумовых, оптимизированных к параметрам сигнала опознавания (наихудших шумовых помех) и внутрисистемных помех. Результаты исследования позволяют выбирать сигнально-кодовые конструкции с ШПС, обеспечивающие наиболее высокую помехоустойчивость для конкретной помеховой обстановки. 


\section{Список литературы}

[1] Ширман Я.Д., Багдасарян С.Т., Лосев Ю.И. и др. Радиоэлектронные системы:: Ocновы построения и теория: справочник. М.: Радиотехника, 2007, 512 с. [Shirman Ya.D., Bagdasaryan S.T., Losev Yu.I. et al. Radio-electronic systems: bases of creation and theory: reference book. M.: Radiotekhnika, 2007, 512 p. (in Russian)].

[2] Мелихов Ю.Н., Фролов Д.В., Шевчук В.И. Метод согласованного применения имитостойких широкополосных сигналов во вторичных радиолокаторах. Радиотехника, 2010, 11 [Melikhov Yu.N., Frolov D.In., Shevchuk V.I. Metod of the coordinated application of imito-resistant broadband signals in secondary radars, Radiotekhnika, 2010, 11 (in Russian)].

[3] Иванов А.Н., Кузьмин В.Г., Мурахленко А.С., Шевчук В.И., Ягольников С.В. Оценка эффективности и оптимизация параметров шумовых импульсных помех линиям радиосвязи с временным разделением каналов и шумоподобными сигналами. Радиотехника, 1996, 1, 44-47 [Ivanov A.N., Kuzmin V.G., Murakhlenko A.S., Shevchuk V.I., Yagolnikov S.V. Assessment of efficiency and optimization of parameters of noise impulse interference to lines of a radio communication timedivision channels and noise-type signals, Radiotekhnika, 1996, 1, $44-47$ (in Russian)].

[4] Джангирян С.С., Мелихов Ю.Н., Хмаров И.М., Шевчук В.И. Адаптивный прием сигналов с импульсно-временным кодированием информации в условиях сигналоподобных помех. Радиотехника, 2009, 1 [Dzhangiryan S.S., Melikhov Yu.N., Khmarov I.M., Shevchuk V.I. Adaptive signal pick-up with pulse and time data coding in conditions the signalopodobnykh of noises, Radiotekhnika, 2009, 1 (in Russian)].

[5] Борисов В.И., Зинчук В.М., Лимарев А.Е., Мухин Н.П., Нахмансон Г.С. Помехозащищенность систем радиосвязи с расширением спектра сигналов модуляцией несущей псевдослучайной последовательностью. М.: Радио и связь, 2003, 640 с. [Borisov V.I., Zinchuk V.M., Limarev A.E., Mukhin N.P., Nakhmanson G.S. Noise immunity of the systems of a radio communication with expansion of a range of signals modulation by the bearing pseudorandom sequence. M.: Radio i svyaz, 2003, 640 p. (in Russian)].

[6] Васин В.А., Власов И.Б., Егоров Ю.М. и др. Информаиионные технологии в радиотехнических системах. М.: изд-во МГТУ им. Н.Э. Баумана, 2003, 672 с. [Vasin V.A., Vlasov I.B., Egorov Yu.M., etc. Information technologies in radio engineering systems. M.: izd-vo MGTU im. N.E. Baumana, 2003, 672 p. (in Russian)].

[7] Stark W.E. Coding for coherent frequency-hopped spread-spectrum communication in the presence of jamming, IEEE International Conference on Communications, Conference Record, 1982, 14.2-1-14.2-5.

[8] Howard H. Ma, Margaret A. Poole. Error-Correcting Codes Against the Worst-Case PartialBand Jammer, IEEE Transactions on Communications, 1984, Vol. Com-32(2), 124-133.

[9] Портной С.Л., Жиронкин С.Б. Декодирование блочных кодов в системах передачи информации с псевдослучайным переключением частот. Радиотехника, 1989, 8, 53-56 [Portnoy S.L., Zhironkin S.B. Decoding of block codes in information transmission systems with pseudorandom switching of frequencies, Radiotekhnika, 1989, 8, 53-56 (in Russian)].

[10] Близнюк А.А., Мин Т.А., Петров А.В., Докучаев Я.С. Воздействие наихудшей импульсной помехи на запросный канал системы радиолокационного опознавания с широкополосными сигналами и кодами, исправляющими ошибки. Сборник тезисов «Системы связи и радионави-

$$
-681-
$$


гаиии». Красноярск: АО «НПП «Радиосвязь», 2016, 215-218 [Bliznyuk A.A., Min T.A., Petrov A.V., Dokuchayev Ya.S. Impact of the worst impulse interference on the request channel of a system of radar identification with the broadband signals and codes correcting errors. The Collection of theses of «The communication system and radio navigation». Krasnoyarsk: AO «NPP «Radiosvyaz», 2016, 215-218 (in Russian)].

[11] Варакин Л.Е. Системы связи с шумоподобными сигналами. М.: Радио и связь, 1985, 384 c. [Varakin L.E. Communication systems with noise-type signals. M.: Radio i svyaz, 1985, 384 p. (in Russian)]. 